\title{
Mediastinal Testicular Tumor Compressing the Left Atrium in a Young Male Presenting Initially with Symptoms of Left Heart Failure
}

\author{
Yavuz Pehlivan ${ }^{1}$, Alper Sevinc ${ }^{2}$, Orhan Ozer $^{3}$, Ibrahim Sari $^{3}$ and Vedat Davutoglu ${ }^{3}$
}

\begin{abstract}
Extrinsic left atrial compression is an uncommon source of hemodynamic compromise that can be caused by many mediastinal structures including bronchogenic cysts, carcinoma, lymphoma, thymoma, aortic aneurysm and diaphragmatic hernia. We describe an unusual case of a 26-year-old man presenting with symptoms of left heart failure. Transthoracic echocardiography demonstrated extrinsic left atrial compression caused by a metastatic testis non-seminomatous germ cell tumor. Compression of the left atrium caused impaired left atrial filling, leading to pulmonary venous hypertension and pulmonary edema. To our knowledge, the present case is the first reported case of mediastinal metastatic testis non-seminomatous germ cell tumor causing symptoms of left heart failure by externally compressing the left atrium.
\end{abstract}

Key words: testicular tumor, heart failure, BEP chemotherapy

(Inter Med 48: 169-171, 2009)

(DOI: 10.2169/internalmedicine.48.1623)

\section{Introduction}

Despite the fact that testicular cancer accounts for only $1 \%$ of all man malignancies, it is the most common solid malignancy affecting men between the ages of 15 and 35 years. The management of germ cell tumors of testis has proved to be a model for success among solid tumors. This is basically due to tremendous advances during the past two decades with establishment of cisplatin based chemotherapeutic regimes which have changed the dismal prognosis for patients with advanced disease. Metastases from testicular tumors may have unusual growth and invasive characteristics. For this reason, high volume diseases at atypical locations require surgical procedures (1). We report a patient with left heart failure initially presenting with a nonseminomatous mixed germ cell tumor of the right testis metastasizing into the mediastinum while compressing the left atrium.

\section{Case Report}

A 23-year-old man patient was admitted to the emergency room with complaints of cough, dyspnea, tachypnea and weight loss for the previous month. His symptoms were increased by effort and lying down. His clinical status was compatible with New York Heart Association class III dyspnea. He had lost $10 \mathrm{~kg}$ body weight in the previous 3 months. His medical history was unremarkable, but his mother and elder sister died from lung cancer. The patient had been smoking one pack per day for 4 years. On physical examination, he had distended neck veins, his blood pressure was $150 / 85 \mathrm{mmHg}$ and heart rate was 110 beats/ min. Auscultation revealed cardiac S3 and inspiratory rales in the basal segments of the lungs. In addition to these findings he was found to have a $8 \times 12 \mathrm{~cm}$ firm, nontender right scrotal mass. Laboratory examinations were within normal range except for alpha fetoprotein level (AFP) of more than $300 \mathrm{IU}$ and human choroinic gonadotrophin ( $\beta \mathrm{HCG})$ of

\footnotetext{
${ }^{1}$ Departments of Internal Medicine, Sahinbey Medical Center, Gaziantep University, School of Medicine, Gaziantep, Turkey, ${ }^{2}$ Departments of Medical Oncology, Gaziantep Oncology Hospital, Gaziantep University, School of Medicine, Gaziantep, Turkey and ${ }^{3}$ Departments of Cardiology Sahinbey Medical Center, Gaziantep University, School of Medicine, Gaziantep, Turkey Received for publication August 22, 2008; Accepted for publication September 30, 2008 Correspondence to Dr. Yavuz Pehlivan, drpehlivan@ hotmail.com
} 

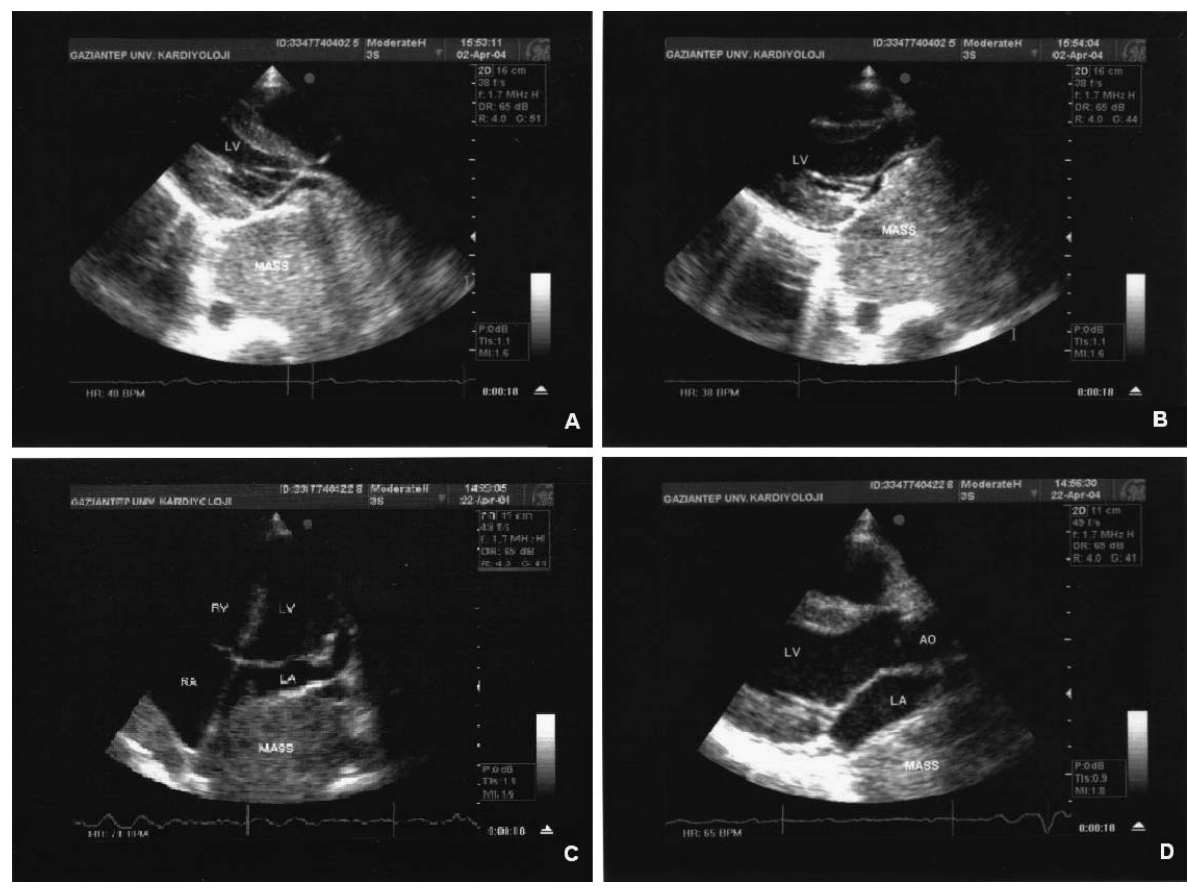

Figure 1. Parasternal long axis views (A and B) of transthorasic echocardiography revealing almost complete obliteration of the left atrium by metastatic mediastinal testicular tumor. Apical four chamber (C) and parasternal long (D) views revealing a dramatic decrease in diameter of the metastatic mass. LV; left ventricle, RV; right ventricle, LA; left atrium, RA; right atrium, AO; aorta

30,417 IU. Computed tomography of thorax revealed one or more heterogenically structured hypodense tumoral lesions which spread from the hilar region to the superior lobe and to the lower basal lobe in the right lung. Moreover, it revealed a hypodense field approximately $5 \mathrm{~cm}$ in diameter around the left atrium. On transthoracic echocardiography a huge, hemorrhagic necrotic mass surrounding and compressing the left atrium and $1 / 3$ of the right atrium was seen (Figs. 1A, 1B). The systolic and diastolic functions were observed to be within normal ranges by echocardiography. There was no other evidence for left heart failure. On scrotal ultrasonography, the left testis was normal. However, a mass filling the right scrotum and extending into the inguinal canal was detected. The mass was $15 \times 8 \times 10 \mathrm{~cm}$ in size with anechoic cystic calcified and vascularized areas. The preliminary diagnosis was testis-derived germ cell tumor which had metastasized to the lung. While waiting for the pathological diagnosis, we started BEP chemotherapy protocol with diuretic treatment for pulmonary edema. Pathological evaluation of the right testis excisional biopsy revealed nonseminamatous mixed germ cell tumor. After the first week of treatment, the patient's clinical condition was improved. At the end of the fourth chemotherapy, tumor markers returned to normal and echocardiography showed dramatic dissappearance of the mass (Figs. 1C, 1D). The patient was referred to the surgery department for operation of the residual tissue.

Discussion

Germ-cell tumor is the most common cancer in young men aged 15-35 years, and is therefore, an extremely important cancer to treat. This testicular tumor spreads in a predictable manner via the lymphatic system to the lymph nodes in the paraaortic chain and then to the supradiaphragmatic nodes in mediastinum and supraclavicular fossa. The most common sites of extranodal disease are the lungs and liver, but spread to the brain and bone is also well recognized (1). It is curable by chemotherapy even in the context of widespread visceral metastases, highly elevated tumor markers (AFP and HCG) and other adverse prognostic features. However, many physicians recommend surgery for disease-free survival in patients with high stage testicular tumor, in whom complete remission could not be achieved with chemotherapy (2).

Germ-cell malignancies arising from mediastium and retroperitoneum represent nearly $2-5 \%$ of all germ-cell malignancies in adults. Other extremely rare extragonadal sites are the central nervous system (pineal, pituitary region, or both), liver and lung. Mediastinal primary ones and absolute refractory ones to first-line cisplatin-based chemotherapy have been identified as among negative prognostic factors. Results of second-line chemotherapy in patients with extragonadal non-seminomatous germ-cell tumor and mediastinal primary site, in particular, are generally inferior to results in testicular non-seminomatous germ-cell tumor (1).

D'Cruz et al (3) divided the relation between mediastinal 
masses and the heart into three categories: proximity (a contiguous or adjacent structure without chamber deformation), encroachment (distortion of normal cardiovascular architexture without hemodynamic effect), and compression. Clinical manifestations of compression include hypotension, hypoxia, tachypnea, and tachycardia as in the present patient. Our patient presented with an unusual cause of pulmonary edema because of extrinsic compression of the left atrium. Possible mechanisms for the observed decreased cardiac output and pulmonary congestion include obstruction of left atrial inflow and direct impairment of ventricular filling leading to functinal tamponade (4). Chorro et al (5) also described a case with extrinsic compression of the right atrium due to a primary mediastinal germ cell tumor.

Transthoracic echocardiography is the test of choice for diagnosing left atrial compression. In contrast to intrinsic atrial abnormalities, such as thrombus or myxoma, extrinsic masses move asynchronously with atria. Transoesophageal echocardiography may also be useful for identification of masses adjacent to left atrium. The other potential important diagnostic tool to disclose the cause of heart failure is thoracic CT. Importantly, thoracic CT might be used not only to determine the interrelation between the mass and cardiac structure but also to identify the etiologic cause of the disease. However, in the consideration of noninvasive and safety properties, transthoracic echocardiography, in our view, should be performed early. In cases of uncertain or doubtful findings additional tools such as thoracic CT should be kept mind.

Extrinsic compression of the left atrium is an unusual cause of congestive heart failure. Other mediastinal structures which might cause left atrial compression are bronchogenic cysts, carcinoma, lymphoma, thymoma, diaphragmatic hernia and commonly aortic aneurysms (6).

In conclusion, this is a rare and unique cause of congestive heart failure due to extrinsic compression of the atrium by a metastatic mediastinal germ cell tumor. Left atrial compression is associated with severe hemodynamic compromise and a quick and accurate diagnosis is of critical importance.

\section{References}

1. May M, Finkbeiner Y, Gunia S, Seehafer M, Knörig J, Hetzer R. Metastasizing testicular germ-cell tumor with infiltration of the right heart: indication for primary metastasectomy. Heart Vessels 21: 63-65, 2006.

2. Tiffany P, Morse MJ, Bosl G, Vaughan ED Jr, Sogani PC, Herr $\mathrm{HW}$, et al. Sequential excision of residual thoracic and retroperioneal masses after chemotherapy of stage III germ cell tumors. Cancer 57: 978-983, 1986.

3. D'Cruz IA, Feghali N, Gross CM. Echocardiographic manifestions of mediastinal masses compressing or encroaching on the heart. Echocardiography 11: 523-533, 1994.

4. Raza ST, Mukherjee SK, Danias PG, Abraham J, Johnson KM,
Sands MJ Jr, et al. Hemodynamically significant extrinsic left atrial compression by gastric structures in the mediastinum. Ann Intern Med 123: 114-116, 1995.

5. Chorro FJ, Cervantes A, Merino J, Solano C, Blanquer J, Wassel A, et al. Extrinsic compression of the right atrium due to a mediastinal germ-cell tumor. Rev Esp Cardiol 44: 553-555, 1991 (in Spanish).

6. Stoupakis G, Fuhrman MA, Dabu L, Knezevic D, Saric M. The use of contrast echocardiography in the diagnosis of an unusual cause of congestive heart failure: achalasia. Echocardiography 21: 149-152, 2004.

(C) 2009 The Japanese Society of Internal Medicine http://www.naika.or.jp/imindex.html 